Published in final edited form as:

Biochemistry. 2008 February 26; 47(8): 2428-2438.

\title{
Characterization of metal binding in the active sites of acireductone dioxygenase isoforms from Klebsiella ATCC 8724
}

\author{
Sergio C. Chai ${ }^{a, f}$, Tingting Jub,e, Marina Dang $^{b}$, Rachel Beaulieu Goldsmith ${ }^{c}$, Michael J. \\ Maroneya , and Thomas C. Pochapsky ${ }^{b, c, d, g}$, \\ aDepartment of Chemistry, University of Massachusetts, Amherst, Massachusetts 01003 \\ bDepartment of Chemistry, MS 015, Brandeis University, Waltham, Massachusetts 02454 \\ cDepartment of Biochemistry, MS 015, Brandeis University, Waltham, Massachusetts 02454 \\ dDepartment of Rosenstiel Basic Medical Sciences Institute, MS 015, Brandeis University, Waltham, \\ Massachusetts 02454
}

\section{Abstract}

The two acireductone dioxygenase (ARD) isozymes from the methionine salvage pathway of Klebsiella ATCC 8724 present an unusual case in which two enzymes with different structures and distinct activities towards their common substrates (1,2-dihydroxy-3-oxo-5-(methylthio)pent-1-ene and dioxygen) are derived from the same polypeptide chain. Structural and functional differences between the two isozymes are determined by the type of $\mathrm{M}^{+2}$ metal ion bound in the active site. The $\mathrm{Ni}^{+2}$-bound NiARD catalyzes an off-pathway shunt from the methionine salvage pathway leading to the production of formate, methylthiopropionate and carbon monoxide, while the $\mathrm{Fe}^{+2}$-bound FeARD' catalyzes the on-pathway formation of methionine precursor 2-keto-4-methylthiobutyrate and formate. Four potential protein-based metal ligands were identified by sequence homology and structural considerations. Based on the results of site-directed mutagenesis experiments, X-ray absorption spectroscopy (XAS) and isothermal calorimetry measurements, it is concluded that the same four residues, His 96, His 98, Glu 102 and His 140, provide the protein-based ligands for the metal in both the Ni- and Fe-containing forms of the enzyme, and subtle differences in the local backbone conformations trigger the observed structural and functional differences between the FeARD' and NiARD isozymes. Furthermore, both forms of the enzyme bind their respective metals with pseudo-octahedral geometry, and both may lose a His ligand upon binding of substrate under anaerobic conditions. However, mutations at two conserved non-ligand acidic residues, Glu 95 and Glu 100, result in low metal contents for the mutant proteins as isolated, suggesting that some of the conserved charged residues may aid in transfer of metal from in vivo sources or prevent the loss of metal to stronger chelators. The Glu 100 mutant reconstitutes readily but has low activity. Mutation of Asp 101 results in an active enzyme that incorporates metal in vivo but shows evidence of mixed forms.

\footnotetext{
* To whom correspondence should be addressed: pochapsk@ brandeis.edu, phone 781-736-2559, fax 781-736-2516.

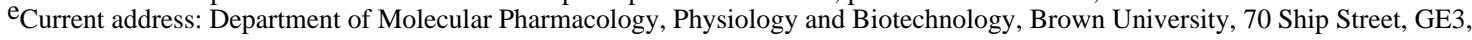
Providence, RI, 02912

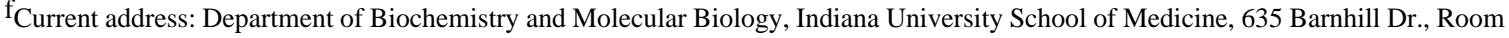
1007, Indianapolis IN 46202

g This work was supported in part by USPHS grant (R01-GM067786 TCP). Acknowledgment is made to the donors of The American Chemical Society Petroleum Research Fund for partial support (MJM). Trainee funding (SCC) was provided by the NIH-Chemistry Biology Interface Program, T32-GM08515. XAS data collection at the National Synchrotron Light Source at Brookhaven National Laboratory was supported by the U.S. Department of Energy, Division of Materials Sciences and Division of Chemical Sciences. Beamline X9B at NSLS is supported in part by the NIH.

Supporting information available

Fits for resting state and ES complex EXAFS data, fit progressions and peak area background corrections are included as supplementary material. This material is available free of charge via the Internet at http://pubs.acs.org.
} 
The methionine salvage pathway (MSP) plays a critical role in regulating a number of important metabolites in prokaryotes and eukaryotes. The first committed intermediate in the MSP, methylthioadenosine (MTA), is formed from $S$-adenosylmethionine (SAM, AdoMet) as a biproduct of polyamine biosynthesis. MTA is a potent inhibitor of polyamine biosynthesis and transmethylation reactions (1). In turn, polyamines are important in the regulation of the cell cycle, being required for cell growth and proliferation. Inhibition of polyamine biosynthesis arrests DNA replication and perturbs cell cycle progression (2), whereas elevated polyamine levels are often associated with tumor formation $(3,4)$. The concentration of MTA is tightly regulated via the MSP, whereby MTA is recycled through a series of reactions that returns the $\gamma$-thiomethyl group of MTA to methionine (5). The penultimate intermediate in this cycle is 1,2-dihydroxy-3-keto-5-methylthiopentene, an acireductone.

In the bacterium Klebsiella ATCC strain 8724, two enzymes have been identified that catalyze the oxidation of acireductone by dioxygen (6). Both of these enzymes are known as acireductone dioxygenases (ARDs) (7). FeARD' converts acireductone to formate and the ketoacid precursor of methionine, 2-keto-4-methylthiobutyrate, whereas acireductone is shunted off-pathway by NiARD to produce 3-methylthiopropionate, carbon monoxide and formate (6-8). This off-pathway shunt has also been observed in Bacillus subtilis (9) and Escherichia coli (10). Both FeARD' and NiARD occur naturally in Klebsiella (10), and both are obtained upon over-expression of a single open reading frame in $E$. coli. Their apoproteins are identical 179-amino acid residue polypeptides with a mass of $20.2 \mathrm{kDa}$, and the activities of the two enzymes are inter-converted by exchanging $\mathrm{Fe}^{+2}$ and $\mathrm{Ni}^{+2}$ (7). Both enzymes are monomeric and co-elute from a size-exclusion column, but can be separated by ion-exchange and hydrophobic interaction chromatography. FeARD' and NiARD represent the only known pair of naturally occurring metalloenzymes with distinct chemical and physical properties determined solely by metal ion content.

Both FeARD' and NiARD are members of the structural superfamily known as cupins (11). A model for the solution structure of NiARD was determined by multidimensional NMR methods (12), and a refinement of that structure recently described (13). X-ray absorption spectroscopy (XAS) studies of the structure of the catalytic Ni center in resting NiARD enzyme and the enzyme-substrate (ES) complex have been reported (14). We have also described a structural model for FeARD' and identified a structural entropy switch that interconverts the two isoforms (15) (Figure 1).

For both the Fe- and Ni-containing isozymes, the paramagnetism of the bound metal complicates the determination of the structure of the active site by NMR methods. For NiARD, the original modeled structure of the active site was based on homology with jack bean canavalin $(12,16)$, and upon refinement, with the recently published crystallographic structure of the house mouse homologue of ARD, MmARD $(13,17)$ (Figure 1). In both cases, the results of XAS studies of NiARD were used to determine Ni-ligand bond lengths and coordination geometry (14). The recently published FeARD' structural model is based on the structure of a stable soluble metal-free mutant of Klebsiella ARD, H98S ARD, after the serendipitous discovery that this mutant is isostructural with FeARD' as determined by multidimensional NMR experiments (15). As with NiARD, Fe-ligand bond lengths and ligation geometry was established using the results of XAS experiments that are detailed here.

Based on the evidence of XAS, site-directed mutagenesis and isothermal calorimetry experiments described in this paper as well as evidence from other laboratories and our own previous work $(14,18)$, we conclude that the same amino acid residues, His 96, His 98, Glu 102 and His 140 provide the protein-based ligands for the metal in both $\mathrm{Fe}$ - and Ni-bound forms of $\mathrm{ARD}$, and that both metals are bound in approximately octahedral geometry, with 
solvent-derived ligands providing the remainder of the coordination sphere. This conclusion implies that relatively subtle differences between the two metal-protein complexes are amplified by the surrounding protein structure, giving two enzymes of different structures and activities from a single polypeptide.

\section{Materials and Methods}

\section{Site-directed mutagenesis, expression and characterization of ARD mutants}

Mutations of proposed metal binding ligands and conserved acidic residues in the ARD active site were generated using the Quik-Change kit and protocol (Stratagene, La Jolla, CA). All primers for mutagenesis were designed in-house and obtained in purified form from Operon Biotechnologies, Inc. (Huntsville, AL). The WT ARD gene in a pET3a vector was used as template (19), and all of the mutant genes were also placed in pET3a expression vectors for amplification and expression. Mutant constructs were amplified in XL1-Blue E. coli (Stratagene), plasmids from selected colonies were then mini-prepped (Promega, Madison, WI) and the presence of the mutations confirmed by complete sequencing of the mutant gene using standard methods (Brandeis University Biotechnology Center).

For protein expression, a pET3a vector containing the appropriate mutant or WT ARD construct was transformed into $E$. coli strain BL21(DE3) and grown in $5 \mathrm{~mL}$ Luria-Bertani (LB) broth containing $50 \mathrm{mg} / \mathrm{L}$ ampicillin. For production of wild type and mutant proteins, 1 $\mathrm{mL}$ of the inoculum culture was transferred to $1 \mathrm{~L}$ of sterile $\mathrm{LB}$ medium containing $50 \mathrm{mg} / \mathrm{L}$ ampicillin. Protein expression was induced when the culture reached a cell density $\mathrm{OD}_{600}$ between 0.5 and 0.6 by the addition of IPTG to a final concentration of $0.5 \mathrm{mM}$. Cells were harvested by centrifugation $3 \mathrm{hrs}$ after induction.

\section{Mutant ARD characterization}

Soluble mutant ARD proteins were purified following previously described methods (19) and protein purity determined by SDS-PAGE gel electrophoresis. For those mutants of ARD found in significant quantities in the cytosol, the metal contents of the purified proteins determined by GF-AAS (Dr. Peter Kerr, Univ. of Massachusetts Amherst). The purified soluble mutants were tested for acireductone dioxygenase activity and compared to WT NiARD and FeARD' by ${ }^{1} \mathrm{H}$ NMR spectroscopy. E100A and H98S mutants were specifically reconstituted with $\mathrm{Ni}^{+2}$ and $\mathrm{Fe}^{+2}$ in the course of ITC experiments (vide infra), and the reconstituted proteins were characterized for keto-acid production from acireductone (on pathway, or FeARD' activity) and $\mathrm{CO}$ production (off-pathway, or NiARD activity). Coupled activity assays have been described previously for production of keto-acid and formate (20). For determination of carbon monoxide production, a gas chromatographic assay was used as described by Sundin (21). In this assay, headspace gas samples released by acidification of enzyme assay solutions are injected via gas-tight syringe onto a polystyrene-divinylbenzene fused silica capillary column $(50 \mathrm{~m} \times 0.32 \mathrm{~mm}$, Agilent catalogue 19091P-Q04) mounted on a Hewlett-Packard model 6890 gas chromatograph operating at $60{ }^{\circ} \mathrm{C}$. Column effluent is mixed with $\mathrm{H}_{2}$ and passed through a $\mathrm{Ni}$ catalyst chamber, so that $\mathrm{CO}$ and $\mathrm{CO}_{2}$ are hydrogenated to methane, then detected by a flame ionization detector. System control and peak quantitation was performed using the Agilent ChemStation software package. Detector response was calibrated by injection of fixed dilutions of $\mathrm{CO}$ gas in $\mathrm{N}_{2}$.

\section{Reconstitution and purification of FeARD'}

In order to insure maximum $\mathrm{Fe}^{+2}$ occupancy of ARD for XAS experiments, WT ARD was reconstituted as follows. WT ARD was expressed as described above, and the cells harvested by centrifugation. $4 \mathrm{~g}$ of cell paste were suspended in $40 \mathrm{~mL}$ of lysis buffer $(50 \mathrm{mM}$ Tris-HCl, pH 8.0, 1 mM DTT, 4 mg DNAase I, 3 mg RNAase A, $100 \mathrm{mg}$ lysozyme, and $10 \mathrm{mg}$ tosyl 
chloride). The mixture was stirred at $4{ }^{\circ} \mathrm{C}$ overnight. All steps up to the chromatography were carried out on ice. The cells were disrupted by sonication ( $70 \%$ output $\times 30 \mathrm{sec} \times 6$ times). Urea (28.8 g) was added while stirring under a stream of argon (Ar) gas to reach a final urea concentration of $8 \mathrm{M}$. DTT was added to a final concentration of $5 \mathrm{mM}$. A Ni ${ }^{+2}$-specific chelator, dimethylglyoxime, was then added to reach a final concentration of $1.4 \mathrm{mM}$. After stirring under $\mathrm{Ar}$ for $10 \mathrm{~min}$, solid $\mathrm{FeSO}_{4}$ was added with stirring to the solution to a final concentration of $10 \mathrm{mM}$. The solution turned slightly green, indicating a minimum degree of $\mathrm{Fe}^{+3}$ formation. After another $10 \mathrm{~min}$, the solution was diluted 8-fold by addition of $420 \mathrm{~mL}$ of degassed and Ar-saturated $50 \mathrm{mM}$ Tris- $\mathrm{HCl}(\mathrm{pH}$ 7.4). The diluted solution was stirred under Ar for an additional $15 \mathrm{~min}$, and then solids were removed by centrifugation (JA-10 rotor, 5000 $\mathrm{rpm}, 60 \mathrm{~min}$ ). The cleared supernatant was loaded onto a DEAE anion exchange column preequilibrated with buffer A ( $20 \mathrm{mM}$ Tris, $\mathrm{pH} 7.4$ degassed and Ar-saturated). After unbound proteins were washed from the column by $100 \mathrm{~mL}$ of buffer $\mathrm{A}$, a linear gradient of $0-0.5 \mathrm{M}$ $\mathrm{NaCl}$ in a total volume of $250 \mathrm{~mL}$ was used to elute FeARD'. ARD fractions were identified by SDS-PAGE gel electrophoresis. The ARD fractions were combined and concentrated, then injected in appropriate amounts onto a Superose 6 size exclusion column equilibrated with 50 $\mathrm{mM} \mathrm{KPi} \mathrm{(pH} \mathrm{7.4)} \mathrm{buffer} \mathrm{and} \mathrm{mounted} \mathrm{on} \mathrm{an} \mathrm{AKTA} \mathrm{FPLC.} \mathrm{Purity} \mathrm{of} \mathrm{fractions} \mathrm{eluted} \mathrm{from} \mathrm{the}$ column was determined by SDS-PAGE. The purest fractions were combined and concentrated for preparation of XAS samples.

\section{XAS Sample Preparation}

Purified resting state FeARD' was buffer-exchanged via desalting column into $50 \mathrm{mM}$ triethanolaminoacetic acid at $\mathrm{pH} 7.5$, and concentrated to $1 \mathrm{mM}$ using a spin concentrator. 50 $\mu \mathrm{L}$ of this solution was loaded into an XAS sample holder and frozen immediately in liquid nitrogen. Due to the relative reactivity of ARD substrate with oxygen, the FeARD' ES complex must be generated in situ anaerobically. The substrate used in this work was a des-thiomethyl form of acireductone, 1,2-dihydroxy-3-oxo-hex-1-ene, produced by the action of E1 enolasephosphatase on the precursor 1-phosphonooxy-2,2-dihydroxy-3-oxo-hexane (22). This substrate shows similar reactivity and product distributions with ARD as the natural substrate (20). Sufficient E1 substrate, $\mathrm{pH}$ adjusted to 7.5, was mixed with FeARD' to obtain a $12 \mathrm{mM}$ final concentration of substrate and $1.6 \mathrm{mM}$ final concentration of FeARD' in $50 \mathrm{mM}$ triethanolaminoacetic acid, $\mathrm{pH}$ adjusted with $\mathrm{NaOH}$ ( $\mathrm{pH}$ 7.5). This solution was allowed to deoxygenate overnight in an anaerobic chamber. Deoxygenated E1 enolasephosphatase and $\mathrm{MgCl}_{2}(30 \mu \mathrm{M}$ and $50 \mu \mathrm{M}$ final concentrations, respectively) were then added to the ARDprecursor mixture and the enolase-phosphatase reaction allowed to proceed for 30 min under anaerobic conditions. The reaction mixture (about $50 \mu \mathrm{L}$ ) was then transferred to the XAS sample holder and immediately frozen in liquid nitrogen.

\section{Preparation of metal-free WT and mutant ARD for isothermal calorimetry experiments}

The procedure used for making metal-free ARD (apo-protein) followed the same protocol described above for preparing reconstituted FeARD' from expression and induction up to the point of dimethylglyoxime addition. Instead of dimethylglyoxime, sodium EDTA (pH 7.4) was added to the urea /DTT solution containing the unfolded ARD to a final EDTA concentration of $10 \mathrm{mM}$. After stirring under Ar for $10 \mathrm{~min}$, the solution was diluted eight-fold with degassed and Ar-saturated $50 \mathrm{mM}$ Tris $\mathrm{HCl} / 10 \mathrm{mM}$ EDTA (pH 7.4). The dilution was stirred under Ar for $15 \mathrm{~min}$, and insoluble material removed by centrifugation $(4000 \times \mathrm{g}, 1 \mathrm{~h})$. The clarified supernatant was loaded onto a DEAE ion exchange column pre-equilibrated with $20 \mathrm{mM}$ Tris, $10 \mathrm{mM}$ EDTA, $\mathrm{pH} 7.4$ (buffer A). After removing unbound proteins with a 100 $\mathrm{mL}$ wash with buffer $\mathrm{A}$, a linear gradient of $0-0.5 \mathrm{M} \mathrm{NaCl}$ in buffer $\mathrm{A}$ over a total volume of $250 \mathrm{~mL}$ was applied to the DEAE column, with the ARD-containing fractions identified by SDS-PAGE gel electrophoresis. These fractions were combined, concentrated and applied to a S-200 size exclusion column pre-equilibrated with buffer A on an AKTA FPLC system. After 
elution as detected by UV detection $(256 \mathrm{~nm})$, the apo-protein was concentrated and dialyzed against $1 \mathrm{~L}$ of $50 \mathrm{mM}$ MOPS, $10 \mathrm{mM}$ EDTA (pH 7.0) for 12 hours, with one buffer change at the six-hour point. EDTA was then removed by dialysis against $50 \mathrm{mM}$ MOPS (pH 7.0) for 36 hours with buffer changes every six hours. Loss of protein was minimized by degassing of all dialysis buffers. The apo-protein was then concentrated appropriately for use in ITC experiments (see below).

\section{Isothermal calorimetry (ITC) titrations for metal binding to WT, H98S and E100A mutant ARD enzymes}

In preparation for ITC experiments, all glassware was washed with $0.5 \mathrm{M} \mathrm{HCl}$ followed by 50 mM EDTA (sodium salt, $\mathrm{pH} 7.5$ ) solution and extensive rinsing with milliQ water. Non-sterile plastic ware was washed with $50 \mathrm{mM}$ EDTA solution followed by extensive rinsing with milliQ water. Sterile plastic ware was considered metal-free and used directly. A 1 M MOPS buffer (sodium salt, $\mathrm{pH}$ 7.0) stock solution was filtered and passed through a Chelex 100 resin column to remove trace metals. All subsequent working buffers were prepared by diluting this stock solution with Chelex-treated milli-Q water. Ultrapure $\mathrm{FeSO}_{4}(99.999 \%)$ and $\mathrm{Ni}\left(\mathrm{NO}_{3}\right)_{2}$ (99.999\%) were purchased from GFS Chemicals (Powell, OH). Metal stock solutions were prepared by dissolving the appropriate amount of salt (measured by weight of the solid) in 50 $\mathrm{mM}$ MOPS pH 7.0. Since $\mathrm{FeSO}_{4}$ is readily air-oxidized, the buffer was degassed for 1 hour under vacuum and then purged with $\mathrm{Ar}$ for 45 minutes before solid $\mathrm{FeSO}_{4}$ was added under an $\mathrm{Ar}$ atmosphere. All subsequent transfer steps for the Fe-containing buffers and samples for Fe titration were made in an $\mathrm{Ar}$ atmosphere. To prevent $\mathrm{Fe}^{+2}$ oxidation, $10 \mathrm{mM}$ sodium dithionite was added to the $\mathrm{FeSO}_{4}$ stock solution (23). Final concentrations for $\mathrm{Ni}$ and $\mathrm{Fe}$ stock solutions were $67 \mathrm{mM}$ and $100 \mathrm{mM}$, respectively. All ITC experiments were performed using a MicroCal VP-ITC microcalorimeter at $25^{\circ} \mathrm{C}$ (Boston Biomedical Research Institute, Watertown, MA). A Hastelloy C-276 sample cell was used for all experiments. ITC titrations were performed at $25{ }^{\circ} \mathrm{C}$ by injection of $\mathrm{nl}$ amounts of the appropriate metal salt in MOPS buffer ( $\mathrm{pH}$ 7.0) into solutions of apo-protein in the ITC cell. Appropriate protein concentrations were determined empirically to provide adequate signal-to-noise in the experiments. All ITC experiments reported were performed at least twice. Data analysis was performed using the Origin software provided with the MicroCal instrument.

\section{XAS Methods}

XAS data for resting state and substrate-bound FeARD' were acquired at beamline X9B at the National Synchrotron Light Source (NSLS) at Brookhaven National Laboratory. Details of XAS data collection and analysis have been recently described for the resting state FeARD' (15). Data collection for the ES complex was performed under identical conditions using a He cryostat to hold the sample temperature near 50K. X-ray fluorescence data were collected using a 13-element Ge detector (Canberra). X-ray absorption data were collected over the range from ca. 6.9-8.1 KeV. Harmonic rejection was achieved by use of a Ni mirror. The average of 10 scans were used for EXAFS analysis by WinXAS (24). The co-added energycalibrated datasets were corrected for background and normalized using two third-order polynomial fits. For XANES analysis, the pre-edge peak at $c a .7114 \mathrm{eV}$ is associated with a $1 \mathrm{~s} \rightarrow 3 \mathrm{~d}$ electronic transition. The area of the peak associated with this transition provides a reliable indication of coordination number/geometry and was determined by fitting a baseline to the pre-edge and edge regions of the spectra ( $c a$. $7105-7120)$ using a cubic function for the pre-edge and $75 \%$ Gaussian and 25\% Lorenzian function to fit the rise in fluorescence occurring at the edge, as previously described (see Supplementary Material) (25). The difference between the fitted background and the spectrum was then integrated. The apparent edge energy of the samples was determined by finding the maximum in the first derivative of the XANES spectrum. 
For EXAFS, the data were converted to $k$ space using the expression $\left[2 m_{e}\left(E-E_{0}\right) / \mathrm{h}^{2}\right]^{1 / 2}$, where $m_{\mathrm{e}}$ is electronic mass, $E$ is the photon energy, $\mathrm{h}$ is Planck's constant divided by $2 \pi$, and $E_{0}$ is the threshold energy of the absorption edge, $7125.0 \mathrm{eV}$. A least-squares fit was employed over a range of $k=2-12.5 \AA^{-1}$. The fitting procedure minimized

$1 / \sigma^{2} \sum_{i=1}^{N}\left[y_{\exp }(i)-y_{\text {theo }}(i)\right]^{2}$, where $\sigma$ is an estimate of the experimental error, $y_{\exp }$ and $y_{\text {theo }}$ are experimental and theoretical data points, respectively, and $N$ is the number of data points (24).

$$
\text { residual }[\%]=\left[\frac{\sum_{i=1}^{N}\left|y_{\exp }(i)-y_{\text {theo }}(i)\right|}{\sum_{i=1}^{N}\left|y_{\exp }(i)\right|}\right] 100
$$

Fits were generated to unfiltered data over the range of $k=2-12.5 \AA^{-1}$ and were limited by noise in the data that was due largely to the dilute nature of the samples and the detector available. Theoretical phases and amplitudes for EXAFS analyses were obtained from calculations of model compounds catena-(hexakis( $\mu$-2-imidazoyl-N-N')bis(imidazole)tri-iron) (26) and $\left(\mathrm{Et}_{4} \mathrm{~N}\right)_{2}\left[\mathrm{Fe}\left(\mathrm{SC}_{6} \mathrm{H}_{4} \mathrm{CH}_{3}-\mathrm{p}\right)_{4}\right]$ using FEFF 8.2 (27). The EXAFS analysis of ES FeARD' data was carried out as described previously (28). Integer values for the number of scattering atoms in a shell were used in the fits without further refinement. Fits were generated using three running parameters for each shell in the first coordination sphere including the distance $(r)$, disorder $\left(\sigma^{2}\right)$ and phase shift $\left(\Delta \mathrm{E}_{0}\right)$. Comparison of the residual (Eq. 1$)$ and the $\sigma^{2}$ parameter were used to select the best fits (24). The values of $\sigma^{2}$ in the best fits are about twice the value expected for a well-ordered shell of scattering atoms. This indicates that either the number of scattering atoms in the shell is too large, or that the site is disordered. Since the number of scattering atoms is determined by the XANES analysis to be six, and since six scattering atoms in the EXAFS fits gave the best residual values, the best fits were selected from those fits containing six scattering atoms in the first coordination sphere (see Supplementary Material). For each shell of scattering atoms in the first coordination sphere, three adjustable parameters were used $\left(r, \sigma^{2}\right.$, and $\left.\mathrm{E}_{0}\right)$. Histidine ligands were "counted" by substituting imidazole ligands for N/O-donor ligands in the first coordination sphere, and using multiple scattering parameters to account for scattering from second coordination sphere $\mathrm{C}$ atoms and third coordination sphere $\mathrm{C}$ and $\mathrm{N}$ parameters, as previously described (28). The value of $\mathrm{E}_{0}$ for $\mathrm{C}$ and $\mathrm{N}$ atoms in the imidazole ring was constrained to be the same as that for the first coordination sphere $\mathrm{N}$-donor atom. This procedure estimates the number of histidine ligands to about $+/-25 \%$, or about the same accuracy with which the number of scattering atoms in the first coordination sphere can be determined from EXAFS analysis alone. A table of selected fits and figures that illustrate the fit progression may be found in the Supplementary Material.

\section{Results}

\section{Site-directed mutagenesis of active site residues in ARD}

$\mathrm{X}$-to-Ala mutations were made for seven residues that are either expected to be metal ligands based on spatial proximity and strong sequence conservation (His 96, His 98, Glu 102 and His 140), have potential for metal ligation with minimal structural distortion (Glu 100), or are conserved or show conservative replacement across the ARD family but are unlikely to provide metal ligands based upon structural considerations (Glu 95, Asp 101) (see Fig. 2). In addition, mutations were made at His $98(\mathrm{H} 98 \mathrm{~S})$ and His $140(\mathrm{H} 140 \mathrm{~F})$ that preserve specific features of the wild-type (polarity at 98 , side-chain planarity at 140). Properties of the mutant proteins are summarized in Table 1. Mutations at three of the proposed ligands His 96, Glu 102 and His 140 all result in decreased expression of soluble protein, with H140A mutant giving no soluble protein. H140F, E102A and H96A all produce some soluble protein but the majority remains in the pellet after cell lysis. 
Mutations at the fourth protein-based ligand, His 98, were of particular interest. The H98A mutant produces a greater fraction of soluble protein than the other three ligand-to-alanine mutants (H140A, H96A and E102A), and the H98S mutant is, like WT, expressed only as soluble protein. We noted that the H98S mutation is iso-structural with FeARD' based upon comparison of multidimensional NMR data, and the structure of H98S ARD was used along with FeARD' XAS data to determine a model for the structure of FeARD' by NMR methods (15). However, H98S ARD exhibits little affinity for either $\mathrm{Ni}^{+2}$ or Fe $\mathrm{Fe}^{+2}$ as measured by ITC titration (see Table 2) indicating that His 98 is likely involved in binding both metals.

The other residue considered as a potential "switch" ligand was Glu 100. Assuming alternating $\beta$-sheet arrangement of amino acid side chains, the side chain of Glu 100 is in register with His 96, His 98 and Glu 102, and like His 98 is also part of the loop between the two outermost strands of the $\beta$-sandwich. With relatively little distortion of the polypeptide, it might be possible for Glu 100 to ligate either metal ion as a fifth protein-based ligand, or act as a replacement for His 98 in one or the other isoform. The E100A mutant is expressed primarily as soluble protein, with an upfield ${ }^{1} \mathrm{H}$ NMR spectrum reminiscent of H98S and FeARD' (Fig. 3), which we have identified as the "default" fold for the ARD polypeptide (15). The E100A mutant has high affinity for both $\mathrm{Ni}^{+2}$ and $\mathrm{Fe}^{+2}$ as determined by ITC experiments, on the same order of magnitude as WT (Table 2), indicating that Glu 100 is not an essential ligand for either metal ion. However, the activities of both the Fe- and Ni-reconstituted E100A mutant are considerably lower than WT enzyme, suggesting that Glu 100 is involved in some manner in enzyme function.

Neither Glu 95 or Asp 101 are expected to ligate the active site metal, since both have their side chains external to the $\beta$-sandwich. However, Glu 95 is strictly conserved in known ARD sequences, and somewhat surprisingly, E95A is expressed primarily as soluble apo-protein (Table 1), with background amounts of Fe present and $\sim 2 \% \mathrm{Ni}$ incorporation. Based on the upfield region of the ${ }^{1} \mathrm{H}$ NMR spectrum the E95A mutant exhibits a perturbed structure resembling that of E102A (Fig. 3). As isolated, E95A shows slight activity above background, but this could be due to the presence of a small amount of active Ni-containing protein.

The upfield ${ }^{1} \mathrm{H}$ spectrum of D101A is complex (data not shown), suggesting multiple forms are present. The D101A mutant as isolated contains significant amounts of both $\mathrm{Ni}$ and $\mathrm{Fe}$. However unlike WT ARD we could not separate distinct Ni- and Fe-containing fractions using either ion-exchange or hydrophobic interaction chromatography. D101A is also the only mutant described here that exhibits significant activity (Table 1) as isolated. While we have not confirmed carbon monoxide production by this mutant, we see $\alpha$-keto-acid formation consistent with ARD' activity.

\section{XANES analysis}

The XANES spectra for resting FeARD' and the FeARD' ES complex are compared in Figure 4. The edge energies determined as the maximum in the first derivative spectrum are 7123.8 (2) $\mathrm{eV}$ for the resting enzyme and 7124.0(2) eV for the ES complex. There is no significant difference in edge energy observed between the resting form and ES complex of FeARD, indicating that no redox change occurs upon substrate binding, as a one-electron metal-centered redox reaction is expected to result in a $2-3 \mathrm{eV}$ shift (25).

At energies near $7113 \mathrm{eV}$, a peak corresponding to a process involving a $1 \mathrm{~s} \rightarrow 3 \mathrm{~d}$ electronic transition is observed. The area under this peak has been shown to be a good measure of the coordination number and geometry in a number of crystallographically characterized iron complexes (29). The peak areas determined for the resting FeARD' and the ES complex are $5.8(1) \times 10^{-2} \mathrm{eV}$ and 6.9(7) $\times 10^{-2} \mathrm{eV}$ respectively, and indicate that the complexes are 6 coordinate in both cases (29-31). 


\section{EXAFS Analysis}

The EXAFS unfiltered and Fourier-transformed spectra for resting FeARD' and the FeARD' ES complex are compared in Figs. 5 and 6, respectively. Visual inspection of the data indicates a change in the spectrum when substrate is added, implying that the substrate binds to the $\mathrm{Fe}$ site. One change that occurs is a decrease in the intensity of features in the Fourier-transformed spectrum at a radial distance of ca. 3-4 $\AA$ (uncorrected for phase shifts) in the spectrum of ES complex. These features are generally attributed to the presence of histidine imidazole ligands; thus, inspection of the spectra indicates either the displacement of a histidine ligand or that a large increase in static disorder leading to an increase of $\sigma^{2}$ occurs upon substrate binding. The curve-fitting analyses of the EXAFS data presented are more consistent with the loss of a histidine ligand upon substrate binding, since the values of $\sigma^{2}$ observed for the first coordination sphere scattering atoms are generally lower (more ordered) in the ES complex, and generally support the qualitative results and provide metric details regarding the Fe sites (see Supplementary Material).

The EXAFS data obtained from resting FeARD' are consistent with a six-coordinate Fe site composed exclusively of $\mathrm{O} / \mathrm{N}$-donor ligands; inclusion of a S-donor ligand leads to poorer fits relative to fits employing $\mathrm{O} / \mathrm{N}$-donors (see Supplementary material). However, as noted in the Experimental section (vide supra) relatively low signal to noise in acquisition and the likelihood of static disorder in the FeARD' active site (as gauged by metal-ligand bond lengths from the crystallographic structure of the MmARD (17)) makes it difficult to clearly identify a single "best-fit" combination of N/O donors and metal-scatterer distances. Comparable fits were obtained with either $\mathrm{O}$ or $\mathrm{N}$-donors, and when the $\Delta E_{0}$ was constrained to a single value for fitting both first and second + third shell scattering atoms or was allowed to vary by atom type ( $\mathrm{N}$ vs. $\mathrm{O}$ ) and shell. The best fit of the data obtained using one value of $\mathrm{E}_{0}$ for $\mathrm{N}$-donors (imidazole ligands) and one $\mathrm{E}_{0}$ for other first coordination sphere donors (O-donors) featured two (N)O-donor ligands at an average distance of $\sim 1.90 \AA$ and four $\mathrm{N}(\mathrm{O})$-donor ligands with an average distance of $\sim 2.06 \AA$, of which three are consistent with His imidazole sidechains when second and third coordination sphere atoms are included: $\mathrm{Fe}(\mathrm{His})_{3}(\mathrm{~N} / \mathrm{O})_{3}$ (Figure 5, see Supplementary Material).

The ligand environment of the Fe center in the ES complex is also best fit by a combination of six N/O-donor ligands. In this case, the fit is consistent with three (N)O-donor ligands at an average distance of $\sim 1.92 \AA$ and three $\mathrm{N}(\mathrm{O})$-donors at an average distance $\sim 2.15 \AA$, of which one of the ligands is a His imidazole sidechain: $\mathrm{Fe}(\mathrm{His})_{1}(\mathrm{~N} / \mathrm{O})_{5}$ (Figure 6, see Supplementary Material). Fits were not improved by including a S-donor. The presence of His $\mathrm{N}$-donor ligands in the FeARD' ES complex is confirmed by multiple scattering analysis. Modeling one of the first coordination sphere $\mathrm{N}$-donor ligands as a His imidazole ligand leads to improvements in the residuals of the fits. However, in this case it is difficult to distinguish whether one or two imidazole ligands are bound.

The crystallographic structure of MmARD shows the metal (presumed but not proven to be $\mathrm{Ni}^{+2}$ ) bound by a 3-His 1-Glu ligation scheme, identical in geometry and sequentially homologous to that proposed in the NMR-derived structures of both NiARD and FeARD' (13,17). The metal-N distances for the three His ligands are $2.02 \AA$ (His 133), $2.05 \AA$ (His 90), $2.19 \AA$ (His 88) and the metal-carboxylate O distance (Glu 94) is $2.2 \AA$. The two remaining sites in the octahedral ligation sphere of the metal not occupied by protein-based ligands are $c i s$ to each other in equatorial positions, and are occupied by an unidentified molecule(s), with N/O ligand distances to the metal of $2.17 \AA$ and $2.25 \AA$. The ligation geometry of the metal is distorted octahedral, with N-Me-N bond angles averaging $97.9^{\circ}$, those between the unknown ligand $(\mathrm{L})$ and the carboxylate $\mathrm{L}-\mathrm{Me}-\mathrm{O}$ averaging $85.5^{\circ}$. The $\mathrm{L}-\mathrm{Me}-\mathrm{L}$ angle between substrate atoms is $72^{\circ}$, and the angle between the ligand carboxylate $\mathrm{O}$ of Glu 94 and the adjacent His $\mathrm{N}$ ligand (His 90 ) is $81^{\circ}$. The $>0.2 \AA$ spread in M-N/O distances $(2.02-2.25)$ 
observed in this structure indicates a high degree of static disorder in the M-L distances and accounts in part for the larger than typical values of $\sigma^{2}$ in the EXAFS models, which were constrained to a maximum of two shells in the first coordination sphere in order to minimize the number of free running parameters in the fits and still accommodate both $\mathrm{N}$ - and $\mathrm{O}$ scattering atoms.

\section{Isothermal calorimetry titrations of WT, H98S and E100A ARD}

Isothermal calorimetry experiments were performed to measure binding of metal ions to three metal-free ARD proteins, WT, E100A and H98S (Table 2). We note that these experiments can only provide an upper limit for $\mathrm{K}_{\mathrm{d}}$ for metal binding because in order to obtain a detectable signal, a minimum of $5 \mu \mathrm{M}$ protein (in the best case, that of WT) is required, putting the upper limit for accuracy in cacluating $\mathrm{K}_{\mathrm{d}}$ at best at $0.05 \mu \mathrm{M}$. Somewhat higher protein concentrations were required for E100A titrations in order to obtain clean isotherms, but strong binding of both $\mathrm{Fe}^{+2}$ and $\mathrm{Ni}^{+2}$ were observed with this mutant. On the other hand, a minimum of $50 \mu \mathrm{M}$ H98S mutant was required in order to detect any heat release above background upon addition of $\mathrm{Ni}^{+2}$, and no reasonable fit could be made to the data. No heat release was observed above background for binding of $\mathrm{Fe}^{+2}$ to $\mathrm{H} 98 \mathrm{~S}$, even at $50 \mu \mathrm{M}$ protein. Furthermore, even when binding of $\mathrm{Fe}^{+2}$ was clearly detected (in the cases of WT and E100A), best fits of binding isotherms gave half-of-the-sites stoichiometry as saturating $\mathrm{Fe}^{+2}$ concentrations, suggesting that $\mathrm{Fe}^{+2}$ binding may drive a dimerization event under the conditions of the ITC experiment (although no evidence for dimerization is observed either by NMR or size-exclusion chromatography for WT FeARD' folded in vivo or by reconstitution for XAS as described above). For these reasons, ITC performed by direct titration does not provide accurate measures for metal binding affinities, and no direct comparison between $\mathrm{Fe}^{+2}$ and $\mathrm{Ni}^{+2}$ binding affinities is possible using the present data. Still, ITC data provides strong evidence for the conclusion that while His 98 is essential to binding both $\mathrm{Fe}^{+2}$ and $\mathrm{Ni}^{+2}$, Glu 100 is not essential in either case.

\section{Discussion}

The four residues that we have identified as ligating the active site metal in both FeARD' and NiARD isoforms, His 96, His 98, Asp 102 and His 140, are all strictly conserved across the ARD superfamily (31), and were initially identified as potential ligands via sequence alignment and spatial localization from paramagnetic broadening patterns in NMR data and homology modeling (12). The recently-published crystal structure of MmARD supports the assignment of these residues as the protein-based ligands (17). However, the identity of the metal in the MmARD structure is unknown, and considering the importance of the identity of the metal in determining both structure and function of ARD, the possibility of alternate metal binding modes involving ligand switching between FeARD' and NiARD could not be ignored. Our primary suspect in this regard was His 98: Unlike His 96, Glu 102 and His 140, which all reside in well-defined $\beta$-structure, His 98 is found on a potentially flexible loop between two strands, and is adjacent to a glycine residue, Gly 99. As such, His 98 seemed the most likely of the four to act as a structural switch between FeARD' and NiARD binding. However, ITC and spectroscopic studies indicate that the H98S mutant does not bind either metal strongly, indicating that $\mathrm{His} 98$ is a ligand for both $\mathrm{Fe}^{+2}$ and $\mathrm{Ni}^{+2}$.

Besides the 3-His 1-Glu ligand tetrad, a number of other acidic residues in the active site region of ARD are either also strictly conserved (Glu 95) or conservatively substituted (Asp for Glu 100, Glu for Asp 101) across the superfamily (32). Of particular interest in this regard is Glu 100. Based on the even registry of the three ligands (His 96, His 98, Glu 102), and its position on the inter-strand loop (Thr 97-His 98-Gly 99-Glu 100-Asp 101) Glu 100 appeared to be structurally well-placed to either provide an extra ligand to the metal with little structural 
distortion or to replace a ligand in one or the other isoform. However, ITC data shows that the E100A mutant binds both $\mathrm{Ni}^{+2}$ and $\mathrm{Fe}^{+2}$ readily and the ${ }^{1} \mathrm{H}$ NMR fingerprint of $\mathrm{Ni}-$ reconstituted E100A ARD is remarkably similar to that of WT NiARD (Fig. 3). Furthermore, the Ni-reconstituted E100A ARD shows CO production from acireductone under standard assay conditions, with no significant $\alpha$-keto-acid production, indicating NiARD activity, while the Fe-reconstituted form gives rise to $\alpha$-keto-acid, indicating FeARD'-like activity. As such, we conclude that Glu 100 is not an essential metal ligand in either ARD isoform. However, we also note that the activities of both reconstituted forms of E100A ARD were much lower than WT (while still maintaining the same metal-dependent chemoselectivity as WT). It is clear that Glu 100 plays a role in the chemistry catalyzed by ARD in both isoforms, and we are currently investigating this function in more detail.

Finally, the roles of the strictly conserved Glu 95 and conservatively substituted Asp 101 must be considered. Neither residue is suitable for direct metal ligation. In all three ARD structures, the carboxylate side chain of Glu 95 (Glu 87 in MmARD) is surface-exposed and its orientation fixed by the register of the $\beta$-helix. In NiARD, the carboxylate of Glu 95 is positioned to interact with portions of the C-terminal peptide (Gly 168-Asp 170) that precedes the C-terminal 3,10helix (13). In MmARD, the homologous Glu 87 forms a surface salt bridge with a nonconserved arginine, $\mathrm{Arg} 108$. The fact that the E95A mutant is isolated primarily as apo-protein, and shows a distorted structure similar to that of a ligand mutant (Fig. 3) suggests that Glu 95 plays a role in metal incorporation in vivo, either by stabilizing the structure of the active site in the apo-protein or assisting in metal transfer from a metallochaperone.

The D101A mutant incorporates metal in vivo and shows the highest enzymatic activity of any of the mutants described here. Structurally, the carboxylate of Asp 101 (conservatively replaced by Glu 93 in MmARD) interacts with a conserved Tyr (Tyr 70 in ARD, Tyr 61 in MmARD) and a conserved positively charged residue at the C-terminal of the E' helix (Lys 68, Arg 59 in MmARD). This region has been implicated in the structural switch between NiARD and FeARD' (15). In NiARD, this region is structurally well-defined and is important in determining the formation of secondary structure in the turn-loop that directs the packing of the C-terminal 3,10 helix onto the top of the $\beta$-sandwich as viewed in Fig. 1. In FeARD', this region occupies multiple conformations on the NMR time scale, and the C-terminal is disordered. This disorder as well as the repacking of the E,E' helices against the bottom of the $\beta$-sandwich have been identified as key structural differences between NiARD and FeARD', and results in a more open active site for ARD' than for ARD (15). Based on the current results, we propose that Asp 101 plays an important role in transmitting local conformational changes in the ARD active site that occur upon Ni binding to other parts of the protein. Current experiments are aimed at clarifying that role.

\section{Comparison of the FeARD' and NiARD resting state and ES complexes by XAS}

Despite the difficulty in obtaining a clear best-fit solution to the EXAFS data, the XAS results allow us to conclude that the structures of the Fe centers in resting FeARD' and in the FeARD' ES complex are strikingly similar to those in the corresponding Ni centers in NiARD and NiARD ES complex (14). The Ni center in resting NiARD was found to be six coordinate with exclusively N/O ligation including 3-4 His imidazole donors: $\mathrm{Ni}(\mathrm{His})_{3-4}(\mathrm{O} / \mathrm{N})_{2-3}$ with one O-donor at $1.91 \AA$ and five N/O donors at $2.10 \AA$. Acireductone was shown to bind to NiARD with the apparent displacement of a histidine, and the predicted NiARD-ES complex consists of four O-donors at a Ni-O distance of $2.00 \AA$ and two N-donors at $2.14 \AA$, with $1-2$ of the ligands being provided by a histidine: $\mathrm{Ni}(\mathrm{His})_{1-2}(\mathrm{O} / \mathrm{N})_{4-5}$. The XAS data thus appear to rule out a change in the type and number of ligand donors or coordination geometry, and do not support the idea that $\mathrm{Fe}$ and $\mathrm{Ni}$ are coordinated to different ligands in the two enzymes. Figure 7 compares the Fourier-transformed spectra for FeARD' and NiARD in the resting state and 
ES complex. The spectra for both ARD proteins are scaled so that the major peak at a radial distance $\sim 2 \AA$ in both spectra have comparable intensities. Both spectra show common features, and are consistent with the binding of the metal ion to the same ligand set in both FeARD' and NiARD. These results are completely consistent with the results from mutagenesis and ITC experiments, both of which support the hypothesis that the same ligand set binds metal in the resting states of NiARD and FeARD'. Furthermore, the data are also consistent with the formation of similar ES complexes for both isoforms.

In the absence of different ligand sets for the two isoforms, we considered the possibility that the different chemistries of NiARD and FeARD' is due to different redox activities of the bound metals. However, nickel in a N/O-donor ligand environment is not expected to have a biologically accessible redox potential (33). Replacement of $\mathrm{Ni}^{+2}$ by $\mathrm{Fe}^{+2}$ could give rise to a mechanism involving activation of $\mathrm{O}_{2}$. However, metal replacement experiments show that FeARD' activity can be partially reconstituted in apo-ARD by addition of $\mathrm{Mg}^{+2}$, another nonredox active metal ion (12). As such, it appears unlikely that the activity differences between the isoforms arise from differences in redox potential. No EPR signal has been observed for FeARD' under aerobic/anaerobic conditions in the presence or absence of acireductone, consistent a divalent Fe center (20) and this oxidation state is supported by the Fe K-edge energies observed for both the FeARD' and the FeARD' ES complex.

\section{Implications for structural and reactivity differences}

A central question still remains: If both the resting state and ES complexes of FeARD' and NiARD have the same overall geometry, and differences in redox chemistry do not enter into consideration, how do binding of $\mathrm{Fe}$ and Ni give rise to different protein structures and different products from the same substrates? We have proposed that relatively small differences between the two metal binding modes could be amplified via a contraction of the ligation sphere around $\mathrm{Ni}^{+2}$ relative to $\mathrm{Fe}^{+2}$ and/or a switch between $\mathrm{N}_{\delta}$ and $\mathrm{N}_{\varepsilon}$ ligation at His 98 (15). Either or both differences could be sufficient to trigger a conformational change in the Thr 97-His 98-Gly 99-Glu 100-Asp 101 inter-strand loop, which in turn could result in the more wide-spread changes that are observed in the switch between NiARD and FeARD'. Intermediate in the proposed trigger is an anti-parallel hydrogen bonding arrangement involving the backbone NHs and carbonyl oxygens of Thr 97 and Ile 163 in the NiARD structure that is inferred from the MmARD crystal structure (Fig. 2) (13). This interaction is clearly missing in the FeARD' structure, as is the salt bridge/polar interactions involving Lys 68, Tyr 70 and Asp 101 discussed above. The Thr 97-Ile 163 backbone hydrogen bond appears to be critical for stabilizing the structure of the $\mathrm{O}$ turn-loop and the P C-terminal helix in NiARD (Fig. 1). The Asp 101-Lys 68-Tyr 70 interaction also likely contributes to stabilizing this structure, as the region from Lys 68-71 loop following the C-terminal of helix E' is disordered in FeARD' (15). The net effect of these differences is to restrict access to the active site in NiARD via side chains that project from loop $\mathrm{O}$ near the metal cluster, particularly the indole of Trp 162. We have proposed a mechanism to account for the different chemistries catalyzed by NiARD and FeARD' based on alternative modes of metal-substrate ligation resulting in Lewis acid activation of either C-2 (in FeARD') or C-3 (in NiARD) towards intramoleuclar nucleophilic attack by peroxide anion $(12,13)$.

The XAS data presented here provide the first detailed structural information regarding Fe binding in the FeARD' ES complex, and help to establish key early steps in reaction mechanism. Because both the resting enzyme and the FeARD' ES complex contain six-coordinate $\mathrm{Fe}$ centers, binding acireductone as a bidentate ligand would require the substitution of two ligands from the Fe center in the resting enzyme. Although we have previously modeled this interaction as resulting from the displacement of the two solvent-derived ligands, comparison of the 
EXAFS data for the resting FeARD' and the ES complex suggests that at least one of these displaced ligands is a histidine. A similar situation is observed in the case of NiARD (14).

Another interesting result of the current work is that even mutations at residues not directly involved in metal ligation (Glu 95 and Glu 100) appear to hamper metal incorporation in vivo (see Table 1). This is particularly obvious from the E100A mutant. While this mutant readily bound both $\mathrm{Fe}^{+2}$ and $\mathrm{Ni}^{+2}$ in vitro with exothermicity comparable to WT, the protein was isolated primarily as apo-protein, with very low nickel content and less than $20 \%$ of the Fe-bound form. This leads us to speculate that the conserved acidic residues in ARD play a role in metal incorporation via transfer from metallochaperones (34). The upfield methyl "fingerprint" of the ${ }^{1} \mathrm{H}$ NMR spectrum is exquisitely sensitive to changes in packing of hydrophobic residues in ARD. Comparisons between NiARD, FeARD' and H98S (15) show that differences in this spectral region are primarily due to perturbations in the packing of the $E$ and $E^{\prime}$ helices against the bottom of the $\beta$-sandwich, which in turn appears to be driven by changes in the vicinity of the active site. It is possible that metal binding by some or all of these residues during folding provides a template around which the active site can properly form, and that in their absence, incorrect forms can accumulate. Certainly, the polypeptide in the vicinity of the ARD active site must be exquisitely sensitive to environment: Even using the same set of ligands, differences in $\mathrm{Fe}$ and Ni binding are sufficient to initiate dramatic structural changes that propagate through much of the molecule external to the $\beta$-sandwich.

\section{Acknowledgments}

The authors thank Dr. Lingyun Rui for help in setting up the CO assay, Dr. Knut Langsetmo (Boston Biomedical Research Institute) for access to their ITC instrument and his generous help in training, Prof. Susan Sondej Pochapsky for help with figures, Ms. Becky Myers for sequencing work and Prof. Lisbeth Hedstrom for helpful discussions.

\section{Abbreviations used}

$\mathrm{ARD}$, acireductone dioxygenase

ATCC, American Type Culture Collection

DEAE, diethylaminoethylcellulose

DTT, dithiothreotol

EDTA, ethylenediaminetetraacetic acid

EXAFS, extended X-ray absorbance fine structure

FPLC, fast protein liquid chromatography

GF-AAS, graphite furnace atomic absorption spectroscopy

IPTG, isopropyl- $\beta$-D-1-thiogalactopyranoside

ITC, isothermal calorimetry

MOPS, 3-morpholinopropanesulfonic acid

MTA, methylthioadenosine

NMR, nuclear magnetic resonance

SAM, S-adenosylmethionine

SDS-PAGE, sodium dodecylsulfate-polyacrylamide gel electrophoresis

XAS, X-ray absorption spectroscopy

\section{References}

1. Schlenk F. Methylthioadenosine. Adv. Enzymol 1983;54:195-265. [PubMed: 6405586]

2. Oredsson SM. Polyamine dependence of normal cell-cycle progression. Biochem. Soc. Trans 2003;31:366-370. [PubMed: 12653640]

3. Marton LJ, Pegg AE. Polyamines as targets for therapeutic intervention. Ann. Rev. Pharmacol. Toxicol 1995;35:55-91. [PubMed: 7598507] 
4. Pegg AE. Polyamine metabolism and its importance in neoplastic growth and as a target for chemotherapy. Cancer Res 1988;48:759-774. [PubMed: 3123052]

5. Shapiro SK, Barrett A. 5-Methylthioribose as a precursor of the carbon chain of methionine. Biochem. Biophys. Res. Comm 1981;102:302-307. [PubMed: 6796086]

6. Wray JW, Abeles RH. The methionine salvage pathway in Klebsiella pneumoniae and rat liver. Identification and characterization of two novel dioxygenases. J. Biol. Chem 1995;270:3147-3153. [PubMed: 7852397]

7. Dai Y, Wensink PC, Abeles RH. One protein, two enzymes. J. Biol. Chem 1999;274:1193-1195. [PubMed: 9880484]

8. Myers RW, Wray JW, Fish S, Abeles RH. Purification and characterization of an enzyme involved in oxidative carbon-carbon bond cleavage reactions in the methionine salvage pathway of Klebsiella pneumoniae. J. Biol. Chem 1993;268:24785-24791. [PubMed: 8227039]

9. Sekowska A, Denervaud V, Ashida H, Michoud K, Haas D, Yokota A, Danchin A. Bacterial variations on the methionine salvage pathway. BMC Microbiol 2004;4

10. Wray JW, Abeles RH. A bacterial enzyme that catalyzes formation of carbon monoxide. J. Biol. Chem 1993;268:21466-21469. [PubMed: 8407993]

11. Dunwell JM, Purvis A, Khuri S. Cupins: the most functionally diverse protein superfamily? Phytochemistry 2004;65:7-17. [PubMed: 14697267]

12. Pochapsky TC, Pochapsky SS, Ju T, Mo HP, Al-Mjeni F, Maroney MJ. Modeling and experiment yields the structure of acireductone dioxygenase from Klebsiella pneumoniae. Nature Structural Biology 2002;9:966-972.

13. Pochapsky TC, Pochapsky SS, Ju T, Hoefler C, Liang J. A refined model for the structure of acireductone dioxygenase from Klebsiella ATCC 8724 incorporating residual dipolar couplings. J. Biomol. NMR 2006;34:117-127. [PubMed: 16518698]

14. Al-Mjeni F, Ju T, Pochapsky TC, Maroney MJ. XAS investigation of the structure and function of $\mathrm{Ni}$ in acireductone dioxygenase. Biochemistry 2002;41:6761-6769. [PubMed: 12022880]

15. Ju T, Goldsmith RB, Chai SC, Maroney MJ, Pochapsky SS, Pochapsky TC. One protein, two enzymes revisited: A structural entropy switch interconverts the two isoforms of acireductone dioxygenase. J. Mol. Biol 2006;363:523-534.

16. Ko TP, Day J, Mcpherson A. The refined structure of canavalin from jack bean in two crystal forms at 2.1 and 2.0 Angstrom resolution. Acta Crystallogr. Sec. D 2000;56:411-420.

17. Xu Q, et al. Crystal structure of acireductone dioxygenase (ARD) from Mus musculus at $2.06 \AA$ resolution. Proteins-Struct. Func. Gen 2005;64:808-813.

18. Hirano W, Gotoh I, Uekita T, Seiki M. Membrane-type 1 matrix metalloproteinase cytoplasmic tail binding protein-1 (MTCBP-1) acts as an eukaryotic aci-reductone dioxygenase (ARD) in the methionine salvage pathway. Genes To Cells 2005;10:565-574. [PubMed: 15938715]

19. Dai Y, Wensink PC, Abeles RH. One protein, two enzymes. J. Biol. Chem 1999;274:1193-1195. [PubMed: 9880484]

20. Dai Y, Pochapsky TC, Abeles RH. Mechanistic studies of two dioxygenases in the methionine salvage pathway of Klebsiella pneumoniae. Biochemistry 2001;40:6379-6387. [PubMed: 11371200]

21. Sundin AM, Larsson JE. Rapid and sensitive method for the analysis of carbon monoxide in blood using gas chromatography with flame ionisation detection. J. Chromatogr. B 2002;766:115-121.

22. Zhang YL, Heinsen MH, Kostic M, Pagani GM, Riera TV, Perovic I, Hedstrom L, Snider BB, Pochapsky TC. Analogs of 1-phosphonooxy-2,2-dihydroxy-3-oxo-5-(methylthio)-pentane, an acyclic intermediate in the methionine salvage pathway: a new preparation and characterization of activity with E1 enolase/phosphatase from Klebsiella oxytoca. Bioorg. Med. Chem 2004;12:38473855. [PubMed: 15210152]

23. Bou-Abdallah F, Arosio P, Santambrogio P, Yang X, Janus-Chandler C, Chasteen ND. Ferrous ion binding to recombinant human $\mathrm{H}$-chain ferritin. An isothermal titration calorimetry study. Biochemistry 2002;41:11184-11191. [PubMed: 12220183]

24. Ressler T. WinXAS: A new software package not only for the analysis of energy-dispersive XAS data. J. Physique IV 1997;7:269-270. 
25. Colpas GJ, Maroney MJ, Bagyinka C, Kumar M, Willis WS, Suib SL, Mascharak P. P.K. X-ray spectroscopic studies of nickel complexes, with application to the structure of Ni sites in hydrogenases. Inorg. Chem 1991;30:920-928.

26. Lehnert R, Seel F. Crystal structure of iron(II) derivative of imidazole. Z. Anorgan. Allgem. Chemie 1978;444:91-96.

27. Kang B, Cai J. Formation and crystal structure of $\left(\mathrm{Et}_{4} \mathrm{~N}\right)_{2}\left[\mathrm{Fe}\left(\mathrm{SC}_{6} \mathrm{H}_{4} \mathrm{CH}_{3}-\mathrm{p}\right)_{4}\right]$. Jiegou Huaxue 1985;4:119-122.

28. Davidson G, Clugston SL, Honek JF, Maroney MJ. An XAS investigation of product and inhibitor complexes of Ni-containing GlxI from Escherichia coli: Mechanistic implications. Biochemistry 2001;40:4569-4582. [PubMed: 11294624]

29. Roe AL, Schneider DJ, Mayer RJ, Pyrz JW, Widom J, Que L. X-Ray absorption spectroscopy of iron-tyrosinate proteins. J. Am. Chem. Soc 1984;106:1676-1681.

30. Randall CR, Zang Y, True AE, Que L, Charnock JM, Garner CD, Fujishima Y, Schofield CJ, Baldwin JE. X-ray absorption studies of the ferrous active site of isopenicillin $\mathrm{N}$-synthase and related model complexes. Biochemistry 1993;32:6664-6673. [PubMed: 8329393]

31. Bertini I, Briganti F, Mangani S, Nolting HF, Scozzafava A. X-Ray absorption studies on catechol 2,3-dioxygenase from Pseudomonas putida Mt2. Biochemistry 1994;33:10777-10784. [PubMed: 8075079]

32. Sauter M, Lorbiecke R, OuYang B, Pochapsky TC, Rzewuski G. The immediate-early ethylene response gene OsARD1 encodes an acireductone dioxygenase involved in recycling of the ethylene precursor S-adenosylmethionine. Plant Journal 2005;44:718-729. [PubMed: 16297065]

33. Maroney MJ. Structure/function relationships in nickel metallobiochemistry. Current Opinion in Chemical Biology 1999;3:188-199. [PubMed: 10226043]

34. Dosanjh NS, Michel SLJ. Microbial nickel metalloregulation: NikRs for nickel ions. Current Opinion in Chemical Biology 2006;10:123-130. [PubMed: 16504569]

35. Kraulis PJ. Molscript - a program to produce both detailed and schematic plots of protein structures. J. Appl. Crystallogr 1991;24:946-950.

\section{Supplementary Material}

Refer to Web version on PubMed Central for supplementary material. 
Ni-ARD

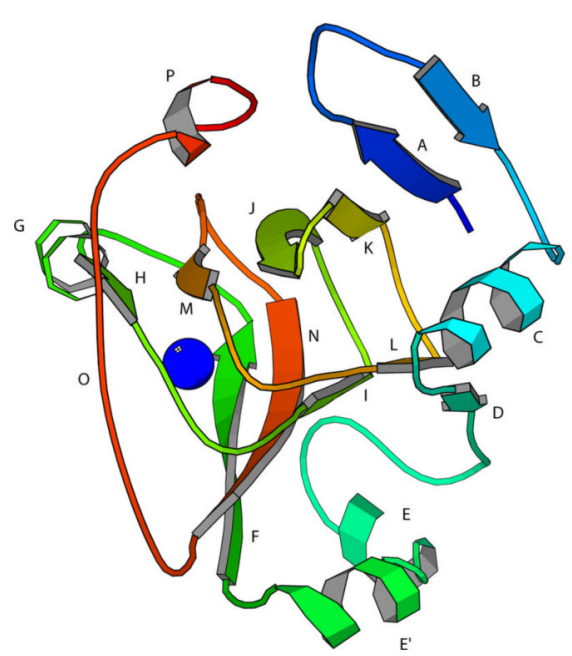

Fe-ARD'

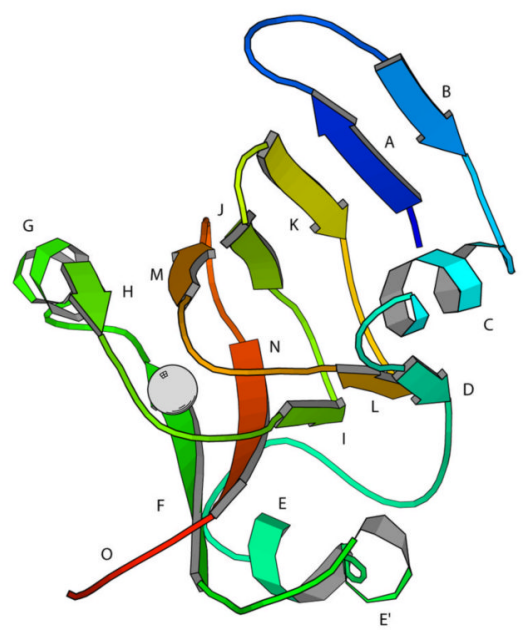

MmARD

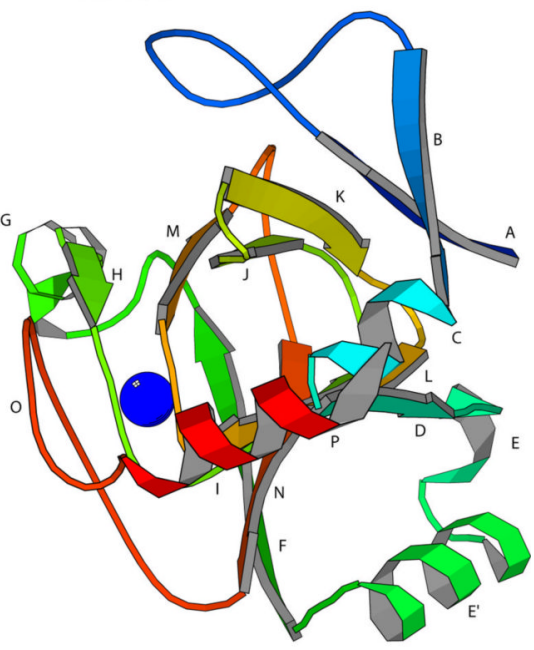

Figure 1.

Solution structures of NiARD (left, PDB entry 1ZRR) and FeARD' (center, PDB entry 2HJI) from Klebsiella and crystallographic structure of MmARD from Mus musculus (right, PDB entry 1VR3). Letters reference to the Klebsiella ARD sequence as follows: A (Ala 2-Phe 6), B (Leu15-Ser18), C (Glu23-Lys31), D (Val33-Glu36), E (Thr52-Tyr57), E' (Ile61-Lys68), F (Ser72-Leu78), G (Lys85-Glu90), H (Phe92-Glu95), I (Arg104-Val107), J (Gly111-Ile117), K (Glu120-Leu125), L (Asn129-Ile132), M (His140-Met144), N (Phe150-Phe156), O (Trp162-Phe166), P (Ile171-Ala174). Features in the MmARD structure are lettered to show correspondence with the Klebsiella ARD structures. Blue spheres show position of $\mathrm{Ni}^{+2}$ in NiARD and unknown metal in MmARD, grey sphere shows position of $\mathrm{Fe}^{+2}$ in FeARD'. Figures were generated using Molscript (35). 


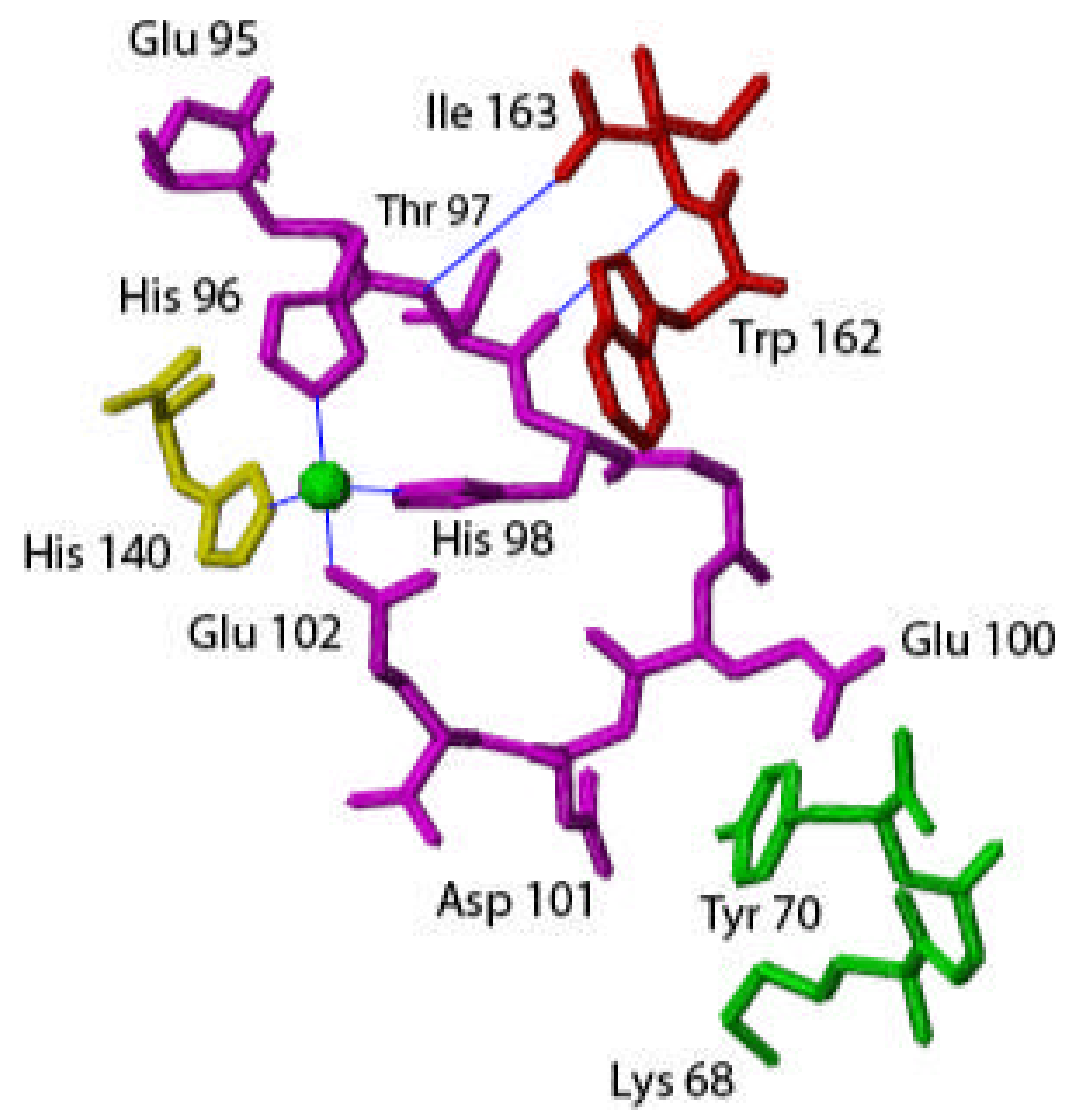

Figure 2.

Details of the metal binding site in NiARD (PDB entry 1ZRR) emphasizing residues mutated in this study and discussed in the text. The nickel is shown as a green sphere. For clarity, the two equatorial water ligands that complete the Ni coordination are not shown. Backbone CONH hydrogen bonds between Thr 97 and Ile 163 and ligand-metal bonds are shown as thin blue lines. View is from the vantage point of the C-terminal end of the G helix (see Figure 1). 

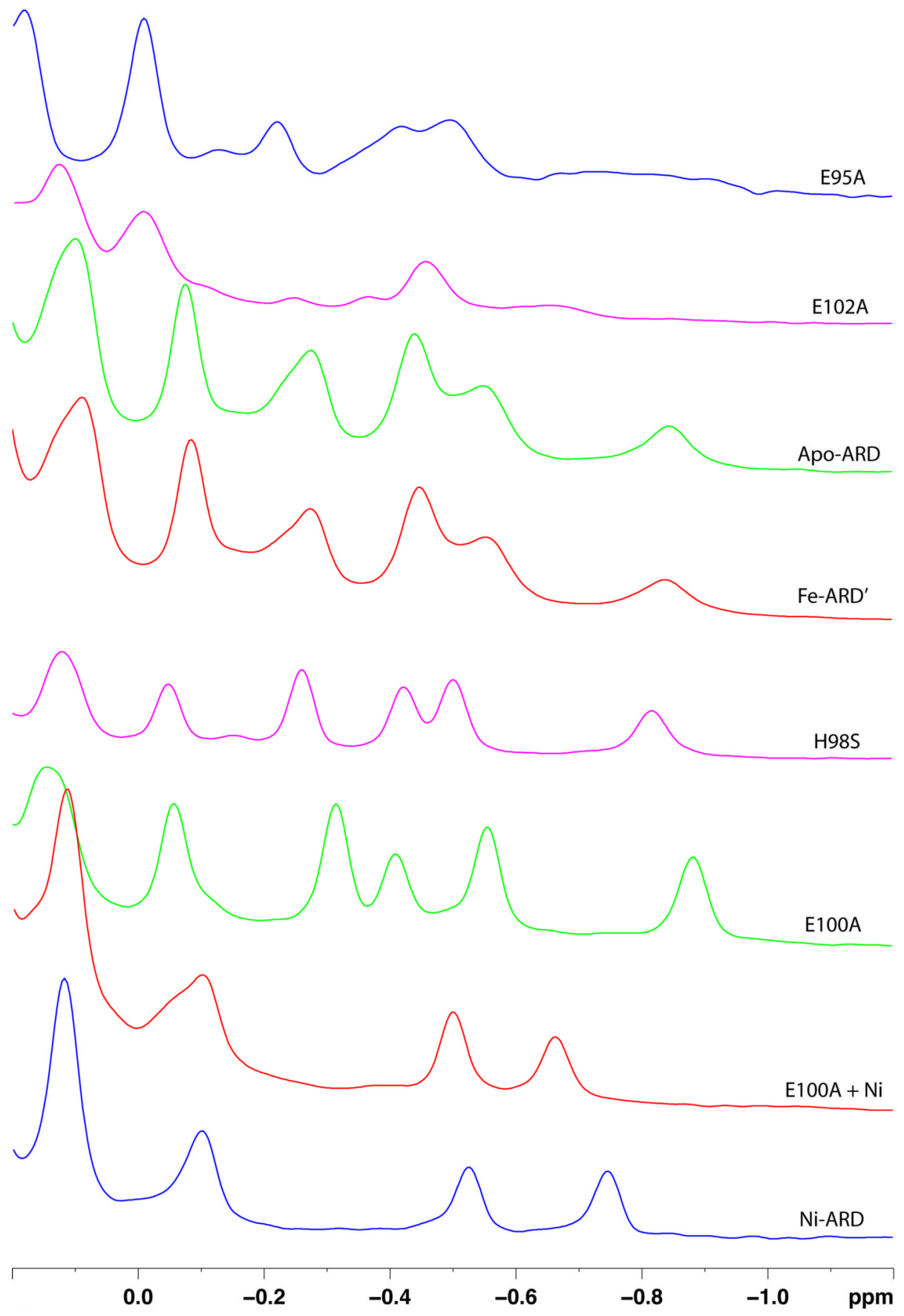

Figure 3.

Comparison of the upfield region of the ${ }^{1} \mathrm{H}$ NMR spectra of various ARD mutants and isoforms. Spectra are arranged so that similarities are emphasized. From top to bottom, E95A as isolated (purple), E102A (carmine), apo-WT ARD (green), FeARD' (red), H98S (carmine), E100A as isolated (green), E100A reconstituted with $\mathrm{Ni}^{+2}$ (red) and $\mathrm{NiARD}$ (purple). All resonances in this region are due to methyl groups in close-packing arrangements with aromatic side chains (15). Note that both E95A and E102A show fewer resonances in this region, indicating a lower degree of order in their structures. ApoARD and FeARD' have very similar folds, as does H98S (15). Apo-E100A shows some similarity to H98S, but after reconstitution with $\mathrm{Ni}^{+2}$ shows a high degree of similarity with NiARD. Detailed resonance assignments of H98S and NiARD 
are available from the BMRB database (www.bmrb.wisc.edu), accession numbers 7103 and 4313 respectively. 


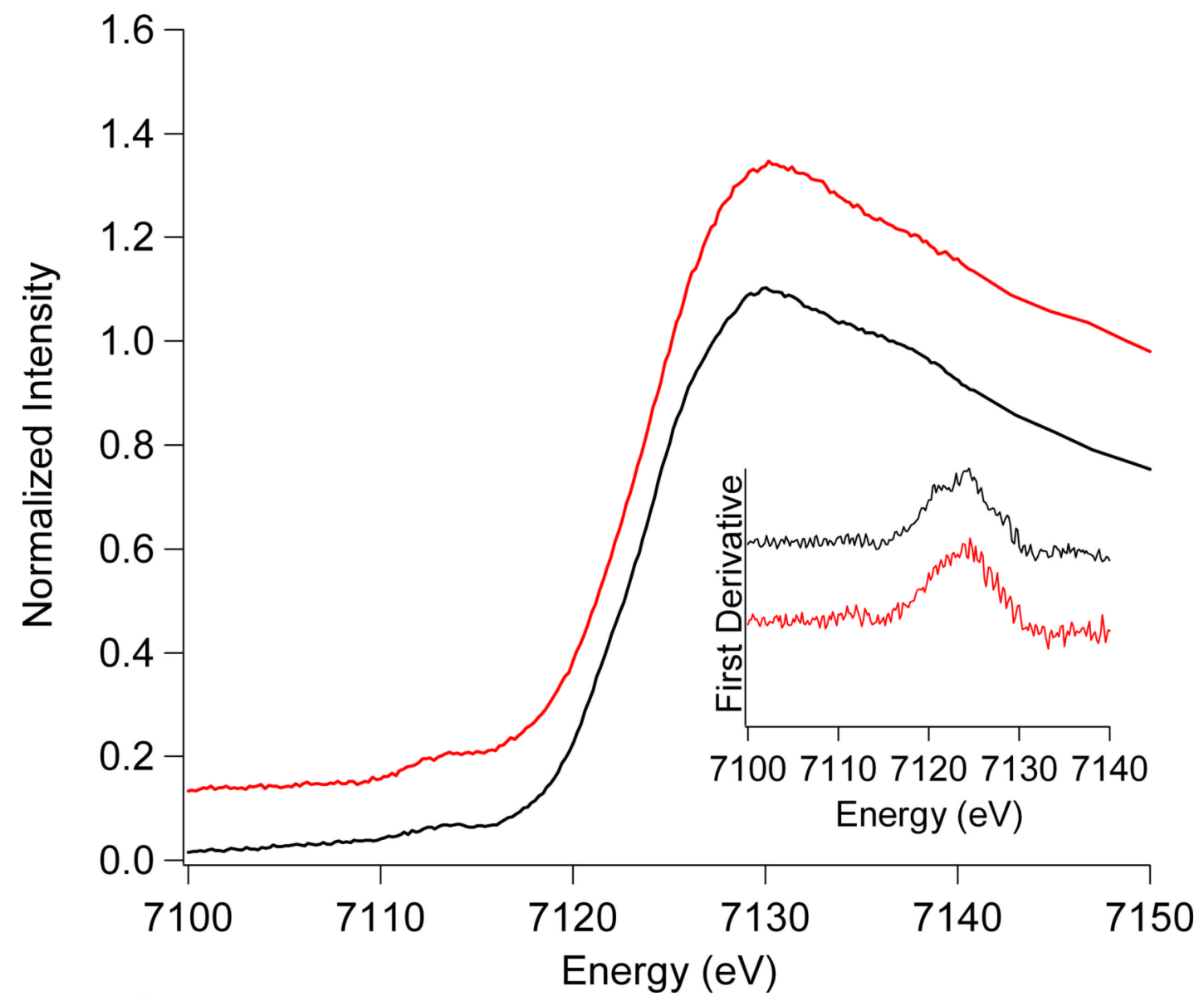

Figure 4.

Fe $K$-edge XANES of the resting state (black) and ES complex (red, offset) of FeARD'. The region around $7114 \mathrm{eV}$ corresponds to the $1 s \rightarrow 3 d$ transition. Insert: First derivative of the XANES in the edge region. 

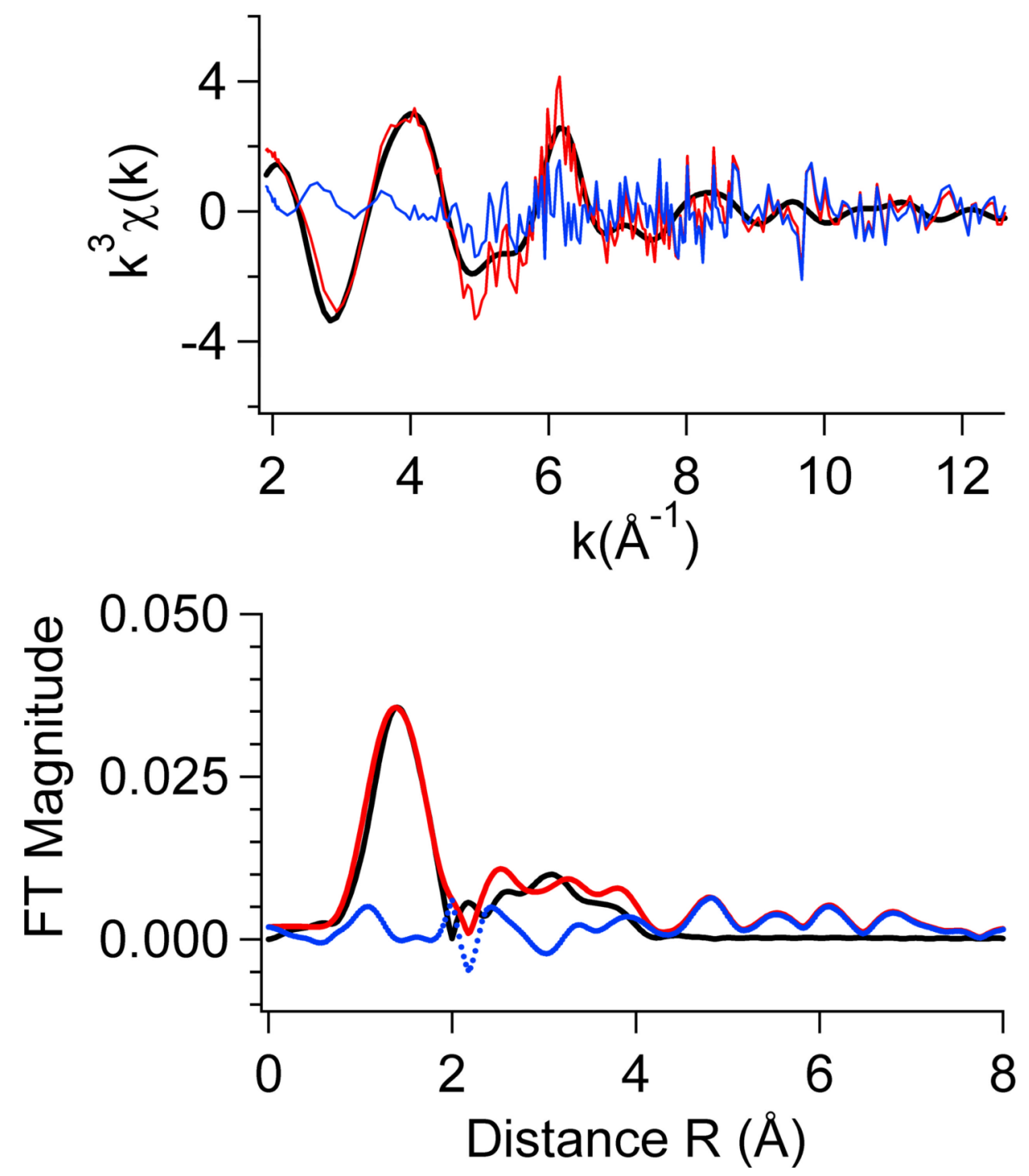

Figure 5.

Fe $K$-edge $\left(k=2-12.5 \AA^{-1}\right)$ EXAFS spectrum for resting FeARD'. Top: $k^{3}$-weighted spectrum (red line) fit (black line) and difference (data-fit; blue line). Bottom: Fourier-transformed data, fit and difference. The fit shown was calculated for $2 \mathrm{O} @ 2.15 \AA+4 \mathrm{~N} @ 1.98 \AA$, including 3 histidine ligands, and corresponds to fit R13 in the supplementary table. 

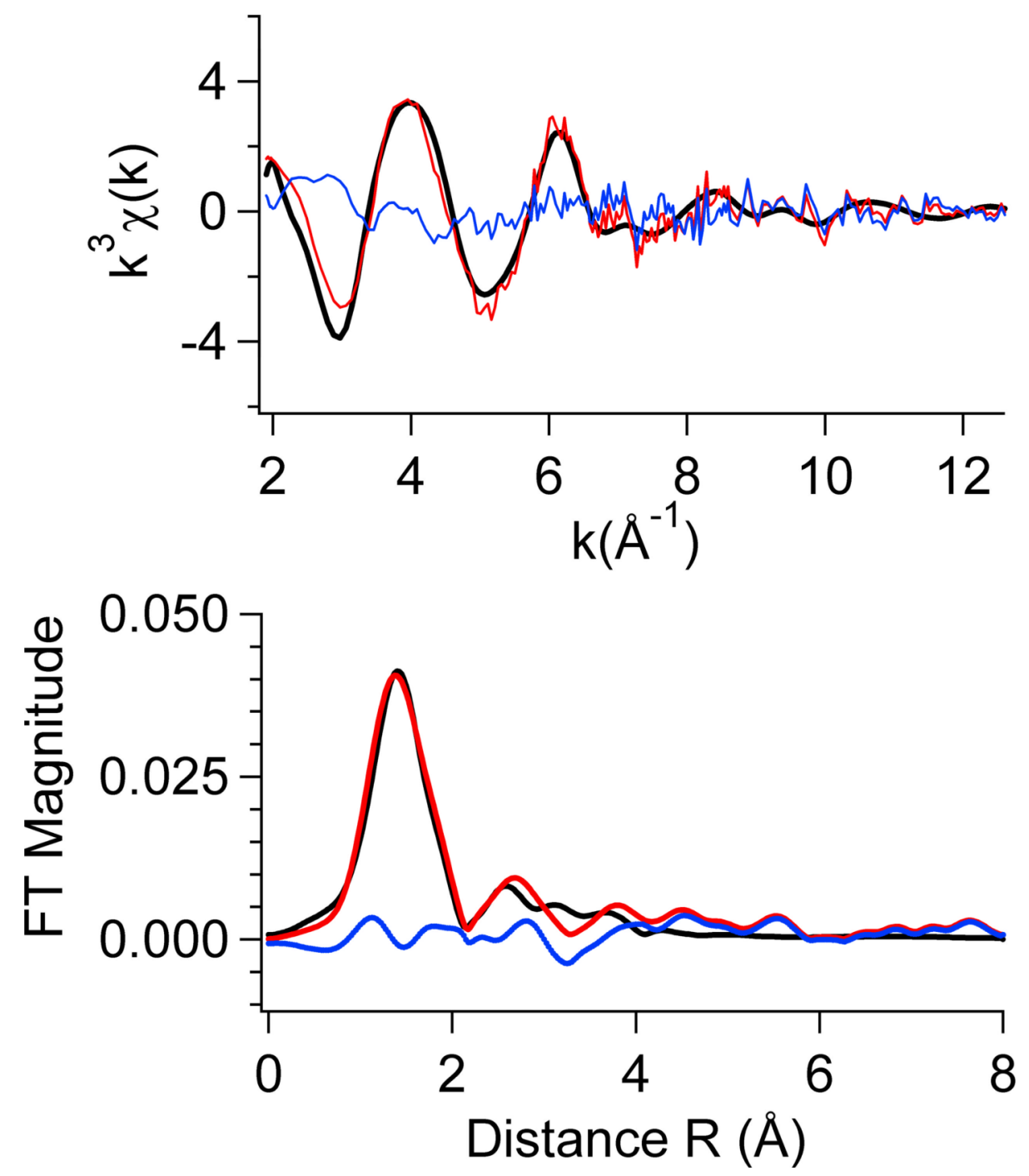

Figure 6.

Fe $K$-edge ( $\left.k=2-12.5 \AA^{-1}\right)$ EXAFS spectrum for FeARD' ES complex. Top: $k^{3}$-weighted spectrum (red line) fit (black line) and difference (data-fit; blue line). Bottom: Fouriertransformed data, fit and difference. The fit shown was calculated for 30 O $2.15 \AA+3 \mathrm{~N} @$ $1.98 \AA$ including 1 histidine ligand and corresponds to fit ES08 in the supplementary table. 

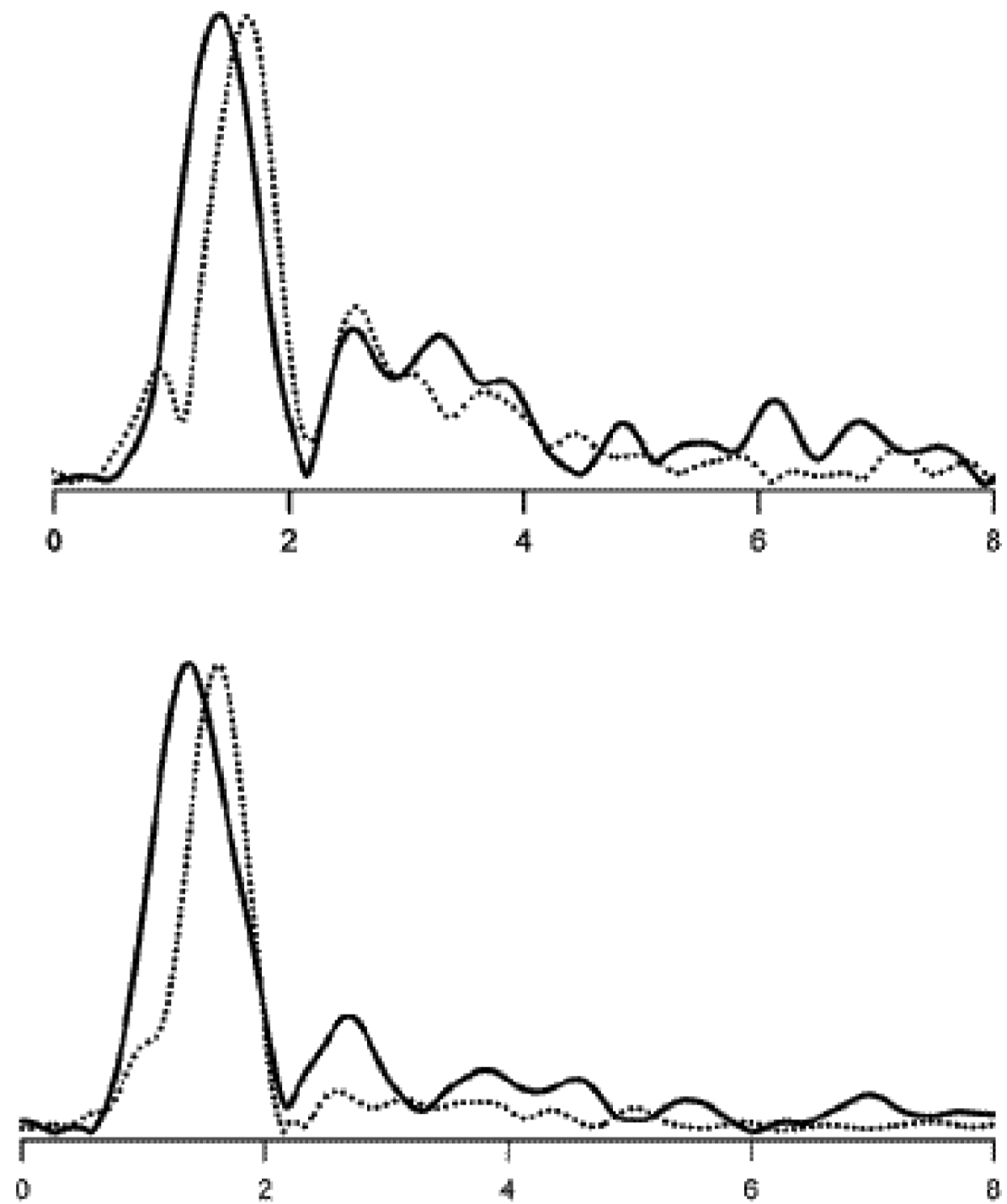

Figure 7.

Comparison of the Fe $K$-edge Fourier-transformed EXAFS spectra $\left(k-2-12.5 \AA^{-1}\right)$ of FeARD' (solid) and Ni $K$-edge EXAFS spectra of NiARD (dashed) of the resting state (top) and ES complex (bottom) from Klebsiella ATCC 8724. 
Characterization of ARD mutants.

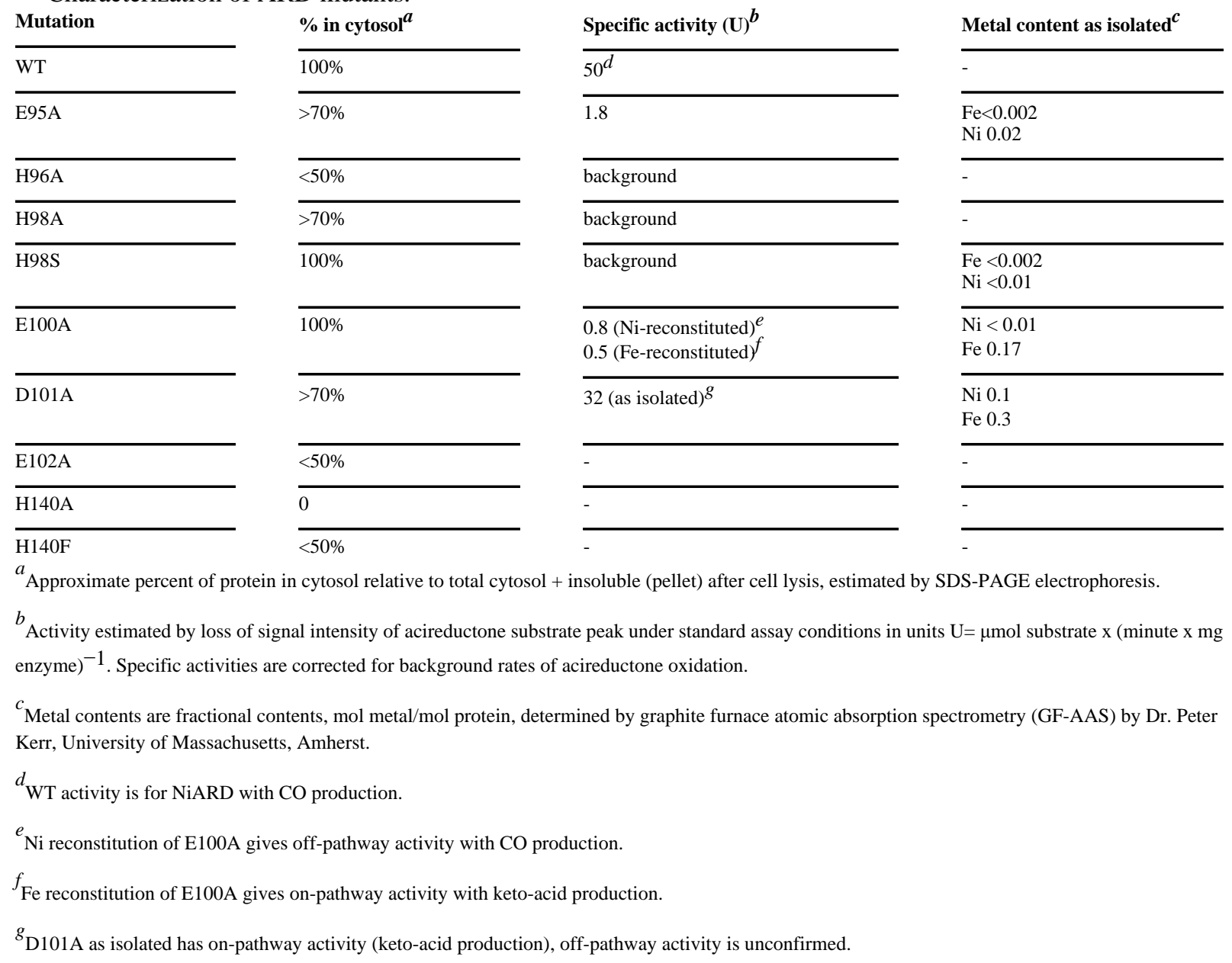

Specific activity $\left(\mathbf{U}^{b}\right.$

Specific activity $(\mathrm{U})^{b}$

\section{Table 1}


Table 2

Isothermal calorimetry results for apo-WT, E100A and H98S ARD. All experimental data sets were best fit to a 1-site model, and all experiments were repeated at least twice. [N] is the stoichiometry ratio (mol metal: mol protein) yielding the apparent $\mathrm{K}_{\mathrm{d}}$. Apparent $\mathrm{K}_{\mathrm{d}}$ values represent upper limits to actual dissociation constants.

\begin{tabular}{|l|l|l|l|l|}
\hline Mutation & $\mathbf{K}_{\mathrm{d}}(\mathbf{a p p}), \mathbf{N i}^{\mathbf{+ 2}}[\mathbf{N}]$ & Protein conc. $(\boldsymbol{\mu M})$. Ni titration & $\mathbf{K}_{\mathrm{d}}(\mathbf{a p p}), \mathbf{F e}{ }^{+2}[\mathbf{N}]$ & Protein conc. $(\boldsymbol{\mu M})$. Fe titration \\
\hline $\mathrm{WT}$ & $\leq 0.1 \mu \mathrm{M}[0.8]$ & 5 & $\leq 0.4 \mu \mathrm{M}[0.5]$ & 25 \\
\hline $\mathrm{H} 98 \mathrm{~S}$ & $>50 \mu \mathrm{M}$ & 50 & $>50 \mu \mathrm{M}$ & 50 \\
\hline $\mathrm{E} 100 \mathrm{~A}$ & $\leq 0.2 \mu \mathrm{M}[0.8]$ & 10 & $\leq 0.2 \mu \mathrm{M}[0.6]$ & 50 \\
\hline
\end{tabular}

